Editorial

\title{
Acknowledgment to Reviewers of Universe in 2020
}

\section{Universe Editorial Office}

Citation: Universe Editorial Office. Acknowledgment to Reviewers of Universe in 2020. Universe 2021, 7, 22. https://doi.org/10.3390/universe702 0022

Published: 22 January 2021

Publisher's Note: MDPI stays neutral with regard to jurisdictional claims in published maps and institutional affiliations.

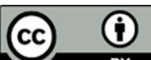

Copyright: (c) 2021 by the author. Licensee MDPI, Basel, Switzerland. This article is an open access article distributed under the terms and conditions of the Creative Commons Attribution (CC BY) license (http://creativecommons.org/licenses /by/4.0/).

MDPI AG, St. Alban-Anlage 66, 4052 Basel, Switzerland

Peer review is the driving force of journal development, and reviewers are gatekeepers who ensure that Universe maintains its standards for the high quality of its published papers. Thanks to the cooperation of our reviewers, in 2020, the median time to first decision was 19 days and the median time to publication was 39 days. The editors would like to express their sincere gratitude to the following reviewers for their precious time and dedication, regardless of whether the papers were published:

Abouelmagd, Elbaz

Abreu, Everton

Acedo, Luis

Addazi, Andrea

Agodi, Clementina

Alberti, Tommaso

Alcolea, Javier

Alexandrovich Zyuzin, Dmitry

Alexeyev, Stanislav

Altschul, Brett

Alvarez-Castillo, David Edwin

An, Tao

Andrew, Keith

Andrianov, Alexander

Andrzejewski, Krzysztof

Anguelova, Lilia

Antonio, Capolupo

Arbuzov, Andrei

Ardenghi, Juan Sebastián

Aros, Rodrigo

Arraut, Ivan

Arzano, Michele

Asada, Hideki

Ashrafi, Nariman

Astashenok, Artyom

Attems, Maximilian

Balbinot, Roberto

Ballardini, Mario

Bamba, Kazuharu

Bambi, Cosimo

Bandos, Igor

Banik, Indranil

Bao, Ning

Barabash, Alexander

Barboza, Edésio Miguel
Barrau, Aurelien

Bartelmann, Matthias

Bastianelli, Fiorenzo

Battistelli, Elia Stefano

Beccaria, Matteo

Beckwith, Andrew

Beltran Jimenez, Jose

Berezin, Victor

Bergeron, Herve

Bermudez, David

Berton, Marco

Bicknell, Geoffrey

Biesiada, Marek

Bietenholz, Wolfgang Peter

Bisnovatyi-Kogan, Gennady

Blaschke, David

Bobomurat, Ahmedov

Boettcher, Igor

Bojowald, Martin

Bolognesi, Stefano

Bonanno, Alfio

Bondarenko, Sergey

Boorman, Peter

Bopp, Fritz

Borges, Humberto

Borovsky, Joe

Bosso, Pasquale

Boulanger, Nicolas

Brack, Andre

Brescia, Massimo

Brizuela, David

Bronnikov, Kirill

Bucciantini, Niccol'o

Bulten, Henk Jan

Burinskii, Alexander 
Cai, Yi-Fu

Caiazzo, Ilaria

Cameron, Robert

Camparo, James

Cao, Zhoujian

Capistrano, Abraao

Capolupo, Antonio

Capozziello, Salvatore

Caracciolo, Vincenzo

Casadio, Carolina

Casadio, Roberto

Casula, Giuseppe

Cavalcanti, Rogerio Teixeira

Cebrián Guajardo, Susana

Celada, Mariano

Chamoun, $\mathrm{N}$.

CHAN, Man

Char, Prasanta

Cheoun, Myung-Ki

Chernodub, Maxim

Chervon, Sergei

Chiarelli, Piero

Chodos, Alan

Choudhury, Sayantan

Chu, Ming Chung

Cicoli, Michele

Cleaver, Gerald

Combrinck, Ludwig

Corasaniti, Pier Stefano

Corda, Christian

Corral, Cristobal

Coughlin, Michael

Cremona, Francesco

Croft, Rupert

Cruz Osorio, Alejandro

Cunha De Holanda, Pedro

Cunha, Pedro

Dabrowski, Mariusz

Dale, Roberto

Danehkar, Ashkbiz

Dasgupta, Arundhati

Davidson, Aharon

De Felice, Antonio

De Leo, Stefano

De Martino, Ivan

De Napoli, Marzio

Debono, Ivan

Degollado, Juan Carlos

Deng, Xue-Mei

Denton, Peter

Deppisch, Frank
Deppman, Airton

Di Felice, Valeria

Dialektopoulos, Konstantinos

Ding, Xuefeng

Dokuchaev, Vyacheslav

Dolgov, Alexander

Done, Chris

Doro, Michele

Drago, Alessandro

Dunne, Gerald

Duong, Minh Quan

Dymnikova, Irina

Edwards, Mark

Edwards, Philip

Efroimsky, Michael

El Moumni, Hasan

Elemér, László

Elizalde, Emilio

Eminov, Pavel

Engel, Jonathan

Erbin, Harold

Escamilla Herrera, Lenin Francisco

Escamilla-Rivera, Celia

Essén, Hanno

Fabrikant, Ilya

Fabris, Júlio

Fabris, Júlio César

Fan, Junhui

Faraggi, Alon

Fatibene, Lorenzo

Fedorova, Elena

Felix, Karbstein

Fendt, Christian

Feoli, Antonio

Ferrer, Efrain

Figueiredo, Cosme Alexandre

Fil'chenkov, Michael

Fomin, Igor Vladimirovich

Foschini, Luigi

Foster, Adam

Frassino, Antonia

Frey, Sandor

Gabuzda, Denise

Gal'tsov, Dmiitry

Gao, Changjun

Gariazzo, Stefano

Garzelli, Maria Vittoria

Gasperini, Maurizio

Gates, James

Gavrilov, Sergey

Gazeau, Jean-Pierre 
Ge, Xianhui

Geng, Chao-Qiang

Georgiev, Danko

Gergely, László

Gharibyan, Hrant

Ghizzo, Alain

Giaccari, Stefano

Giambo, Roberto

Giannoni, Fabio

Gibbons, Gary

Gitman, Dmitry

Giulini, Domenico

Giunti, Carlo

Golterman, Maarten

Gott. III, Richard

Gourgouliatos, Konstantinos

Granemann Souza, Everton

Graziani, Luca

Gregoris, Daniele

Grib, A.

Groote, Stefan

Gubitosi, Giulia

Guendelman, Eduardo

Gulminelli, Francesca

Guo, Feng-Kun

Gusakov, Mikhail

Guzmán, María José

Gyulchev, Galin

Haba, Zbignew

Hadley, Mark

Haggard, Hal

Hamber, Herbert

Han, Wenbiao

Harko, T.

Helayel-Neto, J. A.

Henry, Richard Conn

Herdeiro, Carlos

Holdom, Bob

Hussain, Ibrar

Ingold, Gert-Ludwig

Inui, Norio

Iorio, Lorenzo

Isenberg, Jim

Ivanov, Igor

Javaloyes, Miguel Ángel

Jentschura, Ulrich

Jeong, Donghui

Jetzer, Philippe

Jevicki, Antal

Jipa, Alexandru

Joung, Euihun
Kaempfer, Burkhard

Kalantonis, Vassilis

Kamenshchik, Alexander

Karam, Alexandros

Kassandrov, Vladimir

Katsuragawa, Taishi

Kearns, Ed

Kereszturi, Akos

Ketov, Sergei

Khlopov, Maxim

Khurshudyan, Martiros

Kidonakis, Nikolaos

Kim, Yeongduk

Kimura, Shigeo

Kirillov, Alexander

Kisslinger, Leonard

Koibuchi, Hiroshi

Koldobskiy, Sergey

Kološ, Martin

Konoplya, Roman

Kontar, Eduard

Kopeikin, Sergei

Koshelev, Alexei

Kotanjyan, Anna

Krishnan, Badri

Król, Jerzy

Kullie, Ossama

Kuroyanagi, Sachiko

Kwek, Leong Chuan

Kyutoku, Koutarou

L'Huillier, Benjamin

Lattimer, Jim

Lazarovici, Dustin

Lebiedowicz, Piotr

Lee, Chungchi

Lee, Chung-Chi

Lee, Eun Ah

Leonhardt, Ulf

Leontaris, George

Lepe, Samuel

Lev, F.

Li, Shirley Weishi

Li, Xin

Li, Xinzhong

Li, Yu-Feng

Liegener, Klaus

Lin, Chia-Min

Lin, Chunshan

Lin, Guey-Lin

Link, Jonathan

Liu, Yan 
Livine, Etera

Lobato, Ronaldo

Lobo, Francisco

Lobo, Iarley

Logoteta, Domenico

Longo, Michael

Lopez-Eiguren, Asier

LoSecco, John

Lovell, Mark

Lucini, Biagio

Lukes-Gerakopoulos, Georgios

Lunin, Oleg

Luongo, Orlando

Maestro, Paolo

Maharaj, Sunil

Malek, Emanuel

Maluf Cavalcante, Roberto

Maluf, José Wadih

Mannarelli, Massimo

Marcowith, Alexandre

Mariani, Camillo

Markvorsen, Steen

Marochnik, Leonid

Martín-Albo, Justo

Martino, Ivan De

Martucci, Matteo

Marziani, Paola

Masiello, Antonio

Matsuno, Ken

Mazzitelli, Francisco Diego

McCarroll, Ronald

McCulloch, Mike

Meierovich, Boris

Menendez, Javier

Menezes, Gabriel

Meng, Xin-He

Mertens, Thomas

Messenger, Chris

Michel, Florent

Micol, Benetti

Mielke, Eckehard

Miković, Aleksandar

Millington, Peter

Milton, Kimball

Minamitsuji, Masato

Minkevich, Albert

Miralles, Juan Antonio

Miramonti, Lino

Mizuno, Yosuke

Modanese, Giovanni

Moraes, P.
Morikawa, Masahiro

Mosquera Cuesta, Herman

Mostepanenko, Vladimir

Moustakidis, Charalampos

Mukherjee, Suvodip

Muñoz, Gerardo

Nagai, Hiroshi

Nagirner, Dmitrij

Nampalliwar, Sourabh

Nara, Yasushi

Navarro-Salas, Jose

Nedkova, Petya

Nian, Jun

Nicolas, Chamel

Nikolaevich, Frolov Boris

Nikolic, Hrvoje

Nilsson, Nils Albin

Nishi, Celso

Nojiri, Shin'ichi

Normann, Ben David

Nowakowski, Marek

Nozzoli, Francesco

Nunes, Rafael

O'raifeartaigh, Cormac

Obukhov, Yuri

Odintsov, Sergei

Oikonomou, Vasileios

Okorokov, Vitaly

Olmo, Gonzalo

Otsuka, Masaaki

Overduin, James

Ovgun, Ali

Oyama, Kohichiro

Pacheva, Svetlana

Pagliara, Giuseppe

Pajares, Carlos

Palomares-Ruiz, Sergio

Pan, Jinjin

Papaioannou, Athanasios

Park, Inyong

Pasechnik, Roman

Passante, Roberto

Pavlov, Yu.

Pavón, Diego

Percacci, Roberto

Perez, Alejandro

Pérez-García, M. Ángeles

Perivolaropoulos, Leandros

Perlick, Volker

Peron, Roberto

Petreska, Irina 
Petri, Jerome

Petrov, Albert

Pfeifer, Christian

Piattella, Oliver

Pignalberi, Alessio

Platania, Alessia

Polarski, David

Polonyi, Janos

Poonthottathil, Navaneeth

Potekhin, A.

Pouquet, Annick

Przanowski, Maciej

Pullin, Jorge

Qian, Y.H.

Radinschi, Irina

Raduta, Adriana

Rae, Alastair

Ramirez, Cupatitzio

Rastgoo, Saeed

Ratti, Claudia

Redi, Michele

Ricci, Fulvio

Riffel, Rogemar

Robbins, Daniel

Robertson, Hamish

Rocha, Roldão

Rojas, Juan Efrain

Romatschke, Paul

Roshchupkin, Sergei

Rositsa, Miteva

Rosmej, Frank

Rotondo, Marcello

Rovelli, Carlo

Roy, Indrani

Rueda, Jorge

Ruggiero, Matteo Luca

Ruiz, Daniel

Saa, Alberto

Saavedra, Joel

Sabin, Carlos

Sagun, Violetta

Saharian (Saharyan), Aram Azati

Said, Jackson Levi

Saida, Hiromi

Salasnich, Luca

Salewski, Mirko

Salucci, Paolo

Samtleben, Henning

Sanchez, Miguel

Sanchez, Norma

Santopinto, Elena
Santos, J

Satunin, Petr

Savelova, Elena

Savvidy, George

Sazhina, Olga

Scarr, Tzvi

Schmidt, Michael

Schreck, Marco

Sebastian Koivisto, Tomi

Sebastiani, Lorenzo

Shalyt-Margolin, Alexander

Shapiro, Ilya

Sharma, Ram Krishan

Silvi, Pietro

Simkovic, Fedor

Singh, Parampreet

Singleton, Douglas

Sitarek, Julian

Smith, Michael

Smolić, Ivica

Soda, Jiro

Solà Peracaula, Joan

Soma, Arun

Spencer, Edmund

Spyrou, Nikolaos

Staicova, Denitsa

Starobinsky, Alexei

Stavrinos, Panayiotis

Stecker, Floyd

Steene, Griet

Steinheimer, Jan

Stoica, Sabin

Stojkovic, Dejan

Strakovsky, Igor

Struyve, Ward

Sun, Jiarui

Sushkov, Sergei

Sylos Labini, Francesco

Tada, Yuichiro

Teklu, Berihu

Tolley, Andrew

Toroš, Marko

Torrieri, Giorgio

Tsukamoto, Naoki

Tsupko, Oleg

Tumurtushaa, Gansukh

Turko, Ludwik

Ulhoa, Sérgio Costa

Vacca, Gian Paolo

Vagnozzi, Sunny

Van Beveren, Laurens Willems 
Van Holten, J.W.

Vancea, Ion Vasile

Vanhove, Pierre

Vaskonen, Ville

Velhinho, José

Velten, Hermano

Veras, Dimitri

Vereshchagin, Gregory

Vergara, José David

Viaggiu, Stefano

Vignolo, Stefano

Villaescusa-Navarro, Francisco

Vissani, Francesco

Vogt, Ramona

Voicu, Nicoleta

Volkas, Raymond Robert

Voyatzis, George

Waldron, Abbey

Wang, Anzhong

Weber, Fridolin

Wei, Hanyu

Wei, Shao-Wen

Wen, Meng
Wietfeldt, Fred

Wiggins, Brandon

Wlodarczyk, Zbigniew

$\mathrm{Wu}$, Qiang

$\mathrm{Wu}$, Shuoxing

Xie, Fu-Guo

$\mathrm{Xu}$, Jun

$X u$, Renxin

$\mathrm{Xu}, \mathrm{Rui}$

Yakovlev, Dmitry

Yang, Run-Qiu

Yoshida, Shin'ichirou

Yuan, Tianlu

Zabrodin, Evgeny

Zaninetti, Lorenzo

Zaripov, Farkhat

Zhang, Xiangdong

Zhou, Shun

Zhuk, Alexander

Ziaeepour, Houri

Zilhao, Miguel

Zou, Yuanchuan

Zuzel, Grzegorz 